PROCEEDINGS OF THE

AMERICAN MATHEMATICAL SOCIETY

Volume 130 , Number 11, Pages 3177-3184

S 0002-9939(02)06459-6

Article electronically published on March 25, 2002

\title{
REDUCIBILITY MODULO $p$ OF COMPLEX REPRESENTATIONS OF FINITE GROUPS OF LIE TYPE: ASYMPTOTICAL RESULT AND SMALL CHARACTERISTIC CASES
}

\author{
PHAM HUU TIEP AND A. E. ZALESSKIĬ
}

(Communicated by Stephen D. Smith)

\begin{abstract}
Let $G$ be a finite group of Lie type in characteristic $p$. This paper addresses the problem of describing the irreducible complex (or $p$-adic) representations of $G$ that remain absolutely irreducible under the Brauer reduction modulo $p$. An efficient approach to solve this problem for $p>3$ has been elaborated in earlier papers by the authors. In this paper, we use arithmetical properties of character degrees to solve this problem for the groups

$$
G \in\left\{{ }^{2} B_{2}(q),{ }^{2} G_{2}(q), G_{2}(q),{ }^{2} F_{4}(q), F_{4}(q),{ }^{3} D_{4}(q)\right\}
$$

provided that $p \leq 3$. We also prove an asymptotical result, which solves the problem for all finite groups of Lie type over $\mathbb{F}_{q}$ with $q$ large enough.
\end{abstract}

\section{INTRODUCTION}

The Brauer reduction of ordinary representations modulo a prime $p$ provides a link between the ordinary and the modular representations. For finite groups $G$ of Lie type in characteristic $p$ both $p$-modular and ordinary representation theory have made remarkable achievements during the last 30 years; cf. L], J]. However, the regularities of the Brauer reduction have been much less understood. The following problem, which is in a sense the simplest one about reduction modulo $p$ of complex representations, still remains open.

Problem 1.1. Determine the complex representations of a finite group of Lie type that remain irreducible under the reduction modulo the defining characteristic.

In TZ1 we have elaborated an approach which combines the machinery of integral representation theory, structure theory of finite groups of Lie type, the DeligneLusztig theory and modular representation theory of algebraic groups. This method allows us to solve Problem 1.1 in the case $p>3$ for the complex irreducible rep-

Received by the editors March 21, 2001 and, in revised form, June 12, 2001

1991 Mathematics Subject Classification. Primary 20C33, 20 C20.

Key words and phrases. Finite groups of Lie type, reduction modulo $p$, Steinberg representation.

The first author was partially supported by the NSF grant DMS-0070647 and by a research award from the College of Liberal Arts and Sciences, University of Florida.

The authors are grateful to Professor J. G. Thompson and Professor B. H. Gross for constant encouragement. The authors are also thankful to the referee for helpful comments on the paper. 
resentations of $G$, which are unramified above $p$, that is, whose character values belong to an unramified above $p$ extension of the rational number field $\mathbb{Q}$. These results also lead to significant progress on important applications. Two of them are considered briefly in [TZ1]; the first one is the Thompson-Gross problem on classifying globally irreducible lattices $[\mathrm{T}, \mathrm{Gr}$, and the second one is about lifting representations over $\mathbb{Z} / p \mathbb{Z}$ to representations over $\mathbb{Z} / p^{k} \mathbb{Z}$.

In the sequel [TZ2] to TR1], we classify finite connected reductive groups for which all complex representations are unramified. In particular, the results of TZ1] and TZ2] solve Problem 1.1 for finite groups of Lie type $\mathcal{G}^{F}$, where $\mathcal{G}$ is a connected reductive algebraic group in characteristic $p>3, F$ a Frobenius map on $\mathcal{G}$, provided one of the following conditions holds:

(i) the absolute value of every eigenvalue of $F$ is a square and $p$ is a good prime for $\mathcal{G}$;

(ii) $G=G L_{n}(q), U_{n}(q), S L_{2 n+1}(q), S L_{2 n}(q)$ with $n /(2 n, q-1) \in \mathbb{Z}, S U_{2 n+1}(q)$, $S U_{2 n}(q)$ with $n /(2 n, q+1) \in \mathbb{Z}, C S p_{2 n}(q), S O_{n}^{ \pm}(q), C \operatorname{Spin} n_{n}^{ \pm}(q)$ with $q$ odd, $\Omega_{n}^{ \pm}(q)$ with $q$ even, $\operatorname{Spin}_{2 n}^{\varepsilon}(q)$ with $q$ odd and $-\varepsilon=(-1)^{n(q-1) / 2},{ }^{3} D_{4}(q)$; or

(iii) $G=G_{2}(q), F_{4}(q), E_{6}(q),{ }^{2} E_{6}(q)$, and $p>3$.

(Here, $C \operatorname{Sp}_{2 n}(q)$ is the conformal symplectic group, and $C \operatorname{Spin}_{n}^{ \pm}(q)$ is the so-called special Clifford group.)

The main method of TZ1, however, cannot be applied to ramified representations which exist for certain finite groups of Lie type (even though the majority of complex representations of any given finite group of Lie type are unramified). In this paper, we prove an asymptotical result, cf. Theorem 2.2, which solves Problem 1.1 for all finite groups of Lie type over $\mathbb{F}_{q}$ in any characteristic, provided that $q$ is large enough.

We also obtain some results on Problem 1.1] in the case of characteristic 2 and 3.

Theorem 1.2. Let $G$ be one of the following groups: ${ }^{2} B_{2}(q)$ with $q>2,{ }^{2} G_{2}(q)$ with $q>3, G_{2}(q)$ with $q>2,{ }^{2} F_{4}(q)$ with $q>2, F_{4}(q)$ with $q$ even, or ${ }^{3} D_{4}(q)$ with $q>2$ even. Suppose that $\Theta$ is a nontrivial complex irreducible representation of $G$ that remains absolutely irreducible modulo $p$, where the prime $p$ divides $q$. Then $\Theta$ is the Steinberg representation of $G$.

The simple groups ${ }^{2} B_{2}(8), G_{2}(3), G_{2}(4)$, and $F_{4}(2)$ have exceptional Schur multiplier. Their universal covers, and also ${ }^{2} F_{4}(2)^{\prime}$ and ${ }^{3} D_{4}(2)$, have additional complex representations that are irreducible modulo $p$ but not of $p$-defect 0 ; cf. $\$ 3$.

The main idea that we exploit in this paper is to use arithmetical properties of character degrees of finite groups of Lie type.

Throughout the paper, "irreducible" means absolutely irreducible. $\mathbb{P}$ is an algebraically closed field of characteristic $p$. $\operatorname{Irr}(G)$, resp. $\operatorname{IBr}_{p}(G)$, denotes the set of all irreducible complex characters, resp. of irreducible Brauer characters in characteristic $p$, of a finite group $G .|G|_{p}$, resp. $|G|_{p^{\prime}}$, denotes the $p$-part, resp. the $p^{\prime}$-part of $|G|$. If $\mathcal{G}$ is a simple algebraic group defined over $\mathbb{P}$, then $\mathcal{G}(q)$ denotes the fixed point subgroup $\mathcal{G}^{F}$ for a Frobenius endomorphism $F$ on $\mathcal{G}$ with eigenvalues of absolute value $q$, except for the Suzuki and the Ree groups. In the latter case, to avoid irrational numbers, we use $q$ to indicate the order of the standard realization field, so the groups are ${ }^{2} B_{2}(q),{ }^{2} G_{2}(q)$, and ${ }^{2} F_{4}(q)$, where $q=2^{2 a+1}, 3^{2 a+1}$, and $2^{2 a+1}$ for some integer $a \geq 0$, respectively. 
TABLE I. Large prime divisors of character degrees.

\begin{tabular}{|c||c|l|}
\hline$G$ & $h$ & List of $q_{d}$ \\
\hline$S L_{n}(q), n \geq 2$ & $n$ & $q_{n-1}, q_{n}$ \\
$S U_{n}(q), n \geq 3,2 \nmid n$ & $n$ & $q_{n-1}, q_{2 n-4}, q_{2 n}$ \\
$S U_{n}(q), n \geq 4,2 \mid n$ & $n$ & $q_{n-2}, q_{n}, q_{2 n-6}, q_{2 n-2}, q_{2 n}$ \\
$\operatorname{Sp}_{2 n}(q), n \geq 2$ & $2 n$ & $q_{n}, q_{2 n-2}, q_{2 n}$ \\
$\operatorname{Spin}_{2 n+1}(q), n \geq 3$ & $2 n$ & $q_{n}, q_{2 n-2}, q_{2 n}$ \\
$\operatorname{Spin}_{8}^{ \pm}(q)$ & 6 & $q_{3}, q_{4}, q_{6}, q_{8}$ \\
$\operatorname{Spin}_{2 n}^{ \pm}(q), n \geq 4$ & $2 n-2$ & $q_{n}, q_{2 n-4}, q_{2 n-2}, q_{2 n}$ \\
$G_{2}(q)$ & 6 & $q_{2}, q_{3}, q_{6}$ \\
${ }^{2} G_{2}(q)$ & 6 & $q_{2}, q_{6}$ \\
${ }^{2} B_{2}(q)$ & 4 & $q_{1}, q_{4}$ \\
$F_{4}(q)$ & 12 & $q_{6}, q_{8}, q_{12}$ \\
${ }^{2} F_{4}(q)$ & 12 & $q_{4}, q_{6}, q_{12}$ \\
${ }^{3} D_{4}(q)$ & 6 & $q_{3}, q_{6}, q_{12}$ \\
$E_{6}(q)$ & 12 & $q_{8}, q_{9}, q_{12}$ \\
${ }^{2} E_{6}(q)$ & 12 & $q_{8}, q_{12}, q_{18}$ \\
$E_{7}(q)$ & 18 & $q_{12}, q_{14}, q_{18}$ \\
$E_{8}(q)$ & 30 & $q_{20}, q_{24}, q_{30}$ \\
\hline
\end{tabular}

\section{Asymptotical Result}

According to $\left[\mathrm{Zs}\right.$, if $n>2$ and $(p, n) \neq(2,6)$, then $p^{n}-1$ has a primitive prime divisor, that is, a prime divisor that does not divide $\prod_{i=1}^{n-1}\left(p^{i}-1\right)$. In what follows, if $q=p^{f}$, then we denote by $q_{n}$ a primitive prime divisor of $p^{n f}-1$ (if any).

Lemma 2.1. Let $\mathcal{G}$ be a simply connected simple algebraic group defined over $\mathbb{P}$ and let $G=\mathcal{G}(q)$ be a finite group of Lie type with $q=p^{f}$. Let $h$ be the Coxeter number of $\mathcal{G}$. Assume that $q>8$ if $q$ is even, and $q>p$ if $p$ is a Mersenne prime. If $\mathcal{G}$ is of type $A_{1}$, assume $q>64$ if $q$ is even and $q>p^{2}$ if $p$ is a Mersenne prime. Then for any irreducible complex character $\chi$ of $G$, one of the following holds:

(i) $\chi(1)=1$ or $|G|_{p}$.

(ii) $\chi(1)$ is divisible by $q_{d}$ for some $d \geq h / 4$ (and $d \geq h / 3$ if $G \neq{ }^{2} B_{2}(q)$ ).

Proof. The case of twisted groups ${ }^{2} B_{2}(q),{ }^{2} G_{2}(q)$, and ${ }^{3} D_{4}(q)$ can be dealt with directly using the results of $[\mathrm{CR},[\mathrm{DM}],[\mathrm{E}],[\mathrm{EY}]$. Henceforth we assume that $G$ is not isomorphic to any of these three twisted groups. Consider two cases: $\chi$ is unipotent, and $\chi$ is not. In the first case, $\chi(1)$ is the same for $G$ and for the dual group $H:=\mathcal{G}_{a d}(q)$ of adjoint type, and for the latter group the unipotent degrees are listed in [C2, $\S \S 13.8,13.9]$. In the second case, Lusztig's classification of irreducible characters of $G$ (cf. for instance $[\overline{D i M}]$ ) tells us that $\chi$ corresponds to a pair $\left((s), \chi_{u}\right)$, where $(s)$ is a non-central semisimple conjugacy class in $H$ and $\chi_{u}$ is a unipotent character of $C_{H}(s)$, and $\chi(1)=\left(H: C_{H}(s)\right)_{p^{\prime}} \chi_{u}(1)$. The structure of the centralizers of semisimple elements in finite groups of Lie type has been determined in [C1] in the classical case, and in [D], [DL, Sh1], Sh2], and [Sho in the case of exceptional groups. Using these results we can show that $\left(H: C_{H}(s)\right)_{p^{\prime}}$ is divisible by a "big" $q_{d}$. Since the involved calculations are fairly straightforward, we omit them and give the result in Table I. The second column lists the Coxeter 
number of $\mathcal{G}$. If $\chi$ is a unipotent character of $G$ and $\chi(1) \neq 1,|G|_{p}$, or if $\chi$ is a non-unipotent character of $G$, then $\chi(1)$ is divisible by at least one $q_{d}$ from the list given in the third column. The conditions on $q$ guarantee that $q_{d}$ exists for the $d$ 's listed in Table I.

Theorem 2.2. Let $\mathcal{G}$ be a simple algebraic group defined over $\mathbb{P}$ and let $G=\mathcal{G}(q)$ be a finite group of Lie type associated with $\mathcal{G}$, where $q=p^{f}$. Then there is a bound $f_{0}$ depending on the algebraic group $\mathcal{G}$ such that for any $q=p^{f} \geq p^{f_{0}}$ the following hold:

(i) The set of the degrees of complex irreducible characters of $G$ and the set of the degrees of irreducible Brauer characters of $G$ in characteristic $p$ intersect precisely at $\left\{1,|G|_{p}\right\}$.

(ii) If $\chi$ is a complex irreducible character of degree $>1$ and $\chi(\bmod p)$ is irreducible, then $\chi$ is the Steinberg character (multiplied possibly by a character of degree 1).

Proof. A well-known result of C. W. Curtis (cf. [B, Cor. B.5.11] for the simply connected case) states that $\chi \in \operatorname{Irr}(G)$ has $p$-defect 0 if and only if $\chi$ is the Steinberg character (multiplied possibly by a character of degree 1 ), in which case $\chi(1)=|G|_{p}$. Thus (i) implies (ii) and so it suffices to prove (i).

Let $\ell$ be the largest prime divisor (other than $p$ ) of the degrees of the restricted irreducible representations of the simply connected simple group $\mathcal{G}_{s c}$. Let $h$ be the Coxeter number of $\mathcal{G}$ and set $f_{0}=\max \{4 \ell / h, 4\}$. We show that (i) is true whenever $f \geq f_{0}$. Assume the contrary: $f \geq f_{0}$ but there are $\chi \in \operatorname{Irr}(G)$ and $\varphi \in \operatorname{IBr}_{p}(G)$ with $\varphi(1)=\chi(1) \notin\left\{1,|G|_{p}\right\}$.

Let $S=\mathcal{G}_{s c}(q)$ be the finite group of Lie type corresponding to the simply connected group $\mathcal{G}_{s c}$. Then it is known that $K:=O^{p^{\prime}}(G)$ is isomorphic to $S / Z$ for some central subgroup $Z$ of $S$; furthermore, $e:=|G / K|$ divides $q^{2}-1$ and $e \leq h$; cf. for instance GLS. Let $\rho$, resp. $\psi$, be an irreducible constituent of $\left.\chi\right|_{K}$, resp. of $\left.\varphi\right|_{K}$. Since $G / K$ is abelian, $K$ is perfect, and $\chi(1)>1$, we must have that $\rho(1)>1$. Assume that $\rho(1)=|S|_{p}$. Then $\chi(1)$ is divisible by $|S|_{p}=|K|_{p}=|G|_{p}$, whence $\chi$ has $p$-defect zero and so $\chi(1)=|G|_{p}$, a contradiction. Thus $\rho(1) \notin\left\{1,|S|_{p}\right\}$. Clearly, we can view $\rho$ and $\psi$ as characters of $S$. Hence, by Lemma $2.1 \rho(1)$, and so $\chi(1)$, is divisible by $q_{d}$ for some $d \geq h / 4$. But $q_{d}$ divides $p^{q_{d}-1}-1$, so the primitivity of $q_{d}$ implies that $q_{d}-1 \geq d f \geq d f_{0} \geq(h / 4)(4 \ell / h)=\ell$, i.e. $q_{d}>\ell$. By the choice of $\ell$ and by Steinberg's Tensor Product Theorem, $q_{d}$ cannot divide $\psi(1)$. But $q_{d}$ divides $\chi(1)=\varphi(1)$, hence $q_{d}$ must divide $\varphi(1) / \psi(1)$. Since $\varphi$ is a quotient of the induced character $\psi^{G}$, this implies in particular that $q_{d} \leq e$. On the other hand, the primitivity of $q_{d}$ again implies that $q_{d}-1 \geq d f_{0} \geq 4 h / 4=h \geq e$, a contradiction.

Remark 2.3. In the proof of Theorem 2.2 we can actually take $f_{0}=\max \{2 \ell / h, 4\}$. Indeed, if $G \neq{ }^{2} B_{2}(q), G_{2}(q),{ }^{2} G_{2}(q),{ }^{2} F_{4}(q)$, then $d \geq h / 2$ in Lemma 2.1 On the other hand, all the excluded groups are handled in Theorem 1.2 .

\section{Small Characteristic CASE}

The results of [TZ1] do not apply to finite groups of Lie type in characteristic 2 and 3. However, Problem 1.1 for several groups in characteristic 2 and 3 can be settled directly. 
Proposition 3.1. Let $G=G_{2}(q)$ with $q=2^{f}>2$ or $q=3^{f}$, or $G={ }^{2} G_{2}(q)$ with $q=3^{f}>3$. Suppose that a complex irreducible character $\chi$ of $G$ is irreducible modulo $p$, where $p=q^{1 / f}$. Then $\chi$ is either the trivial character or the Steinberg character.

Proof. Assume the contrary: $\chi(\bmod p)$ is irreducible, but $\chi$ is neither the trivial character nor the Steinberg character.

1) First we consider the case $p=3$ and $G=G_{2}(q)$. According to [JLPW], the irreducible 3-modular characters of $G_{2}(3)$ have degree 1, 7, 27, 49, 189 and 729 . By our assumption, $\chi(1)$ is the degree of an irreducible 3-modular character of $G$, hence $\chi(1)=3^{a} 7^{b}>1$ for some $a, b \in \mathbb{Z}^{+}$by Steinberg's Tensor Product Theorem. In particular, $\chi(1)$ is odd. Inspecting the character degrees of $G$ as listed in [E], we see that in this case $\chi(1)$ is divisible by $q^{4}+q^{2}+1$, more precisely, $\chi(1)=d\left(q^{4}+q^{2}+1\right)$ with $d=1, q / 3, q$, or $q^{2}$. This implies that $\chi(1)$ is not a 3 -power. Hence $\chi(1)$ is divisible by 7 , whence 7 divides $q^{4}+q^{2}+1$, i.e. $3 \not f$. But the latter implies that $q^{4}+q^{2}+1$ is divisible by 13 , contrary to the assumption that $\chi(1)=3^{a} 7^{b}$.

2) Next we consider the case $p=3$ and $G={ }^{2} G_{2}(q)$. By our assumption and by Steinberg's Tensor Product Theorem (for the twisted group $G$, cf. [St]), $\chi(1)=3^{a} 7^{b}>1$ for some $a, b \in \mathbb{Z}^{+}$. On the other hand, observe that $\chi(1)$ is divisible either by $(q-1) / 2$ or $q^{2}-q+1$. Choose a primitive prime divisor $\ell$ of $q-1$, resp. of $q^{6}-1$. Then $\ell \neq 3,7$, contrary to the assumption that $\chi(1)=3^{a} 7^{b}$.

3) Finally, let $p=2$. According to JLPW], the irreducible 2-modular characters of $G_{2}(2)=S U_{3}(3) \cdot 2$ have degree 1, 6, 14, and 64 . By our assumption, $\chi(1)=$ $2^{a} 3^{b} 7^{c}>1$ for some $a, b, c \in \mathbb{Z}^{+}$. In particular, $\chi(1)$ is even. Inspecting the character degrees of $G$ as listed in [EY], we see that $\chi(1)$ is one of the following: $q(q \pm 1)\left(q^{4}+q^{2}+1\right), q^{3}\left(q^{3}+\varepsilon\right), q(q+\varepsilon)\left(q^{3}+\varepsilon\right), q\left(q^{4}+q^{2}+1\right) / 3, q(q \pm 1)^{2}\left(q^{2} \mp q+1\right) / 2$, $q(q \pm 1)^{2}\left(q^{2} \pm q+1\right) / 6, q\left(q^{2}-1\right)^{2} / 3$, with $\varepsilon= \pm 1$ and $q \equiv \varepsilon(\bmod 3)$. The case $f=2$ can be easily handled directly, hence we may assume that $f \geq 3$. Consider a primitive prime divisor $\ell$ of $q^{3}-1$ and a primitive prime divisor $\ell^{\prime}$ of $q^{6}-1$. Clearly, $\ell, \ell^{\prime} \neq 2,3,7$ and $\ell\left|\left(q^{2}+q+1\right), \ell^{\prime}\right|\left(q^{2}-q+1\right)$. Since $\chi$ cannot be divisible by $\ell$ and by $\ell^{\prime}$, we come to the conclusion that $\chi(1)=q\left(q^{2}-1\right)^{2} / 3$. If $f \geq 4$, then $\chi(1)$ is divisible by a primitive prime divisor $\ell^{\prime \prime}$ of $q^{2}-1$, which clearly differs from 2 , 3 and 7 , a contradiction. If $f=3$, then $\chi(1)=q\left(q^{2}-1\right)^{2} / 3=2^{3} 3^{4} 7^{2}$ cannot be written as a product of some 6's, 14 's and 64 's, again a contradiction.

Remark 3.2. Observe that $G_{2}(3)$ has exceptional Schur multiplier $\mathbb{Z}_{3}$. The cover $3 G_{2}(3)$ has 10 faithful characters, 4 of degree 27, 4 of degree 189 and 2 of degree 729 , which are irreducible modulo 3 . Even more, the four characters of degree 27 are irreducible modulo any prime, and they give rise to two globally irreducible representations (in the sense of $[\mathrm{Gr}]$ ) of degree 54 . Similarly, $G_{2}(4)$ has exceptional Schur multiplier $\mathbb{Z}_{2}$. The cover $2 G_{2}(4)$ has a faithful character of degree 4096 , which is irreducible modulo 2 .

Proposition 3.3. Let $G=F_{4}(q)$ with $q=2^{f}$ or $G={ }^{2} F_{4}(q)$ with $q=2^{f}>2$. Suppose that a complex irreducible character $\chi$ of $G$ is irreducible modulo 2. Then $\chi$ is either the trivial character or the Steinberg character.

Proof. Assume the contrary: $\chi(\bmod 2)$ is irreducible, but $\chi$ is neither the trivial character nor the Steinberg character. According to $[\mathrm{V}$, the degree of any irreducible 2-modular character of $F_{4}(2)$ can be written in the form $2^{x} 3^{y} 13^{z} 41^{t}$ for 
some $x, y, z, t \in \mathbb{Z}^{+}$. By our assumption and by Steinberg's Tensor Product Theorem, $\chi(1)=2^{a} 3^{b} 13^{c} 41^{d}>1$ for some $a, b, c, d \in \mathbb{Z}^{+}$.

1) First we consider the case $G={ }^{2} F_{4}(q)$. Observe that $\chi(1)$ is divisible either by $q^{2}+1$, or $q^{4}-q^{2}+1$, or $q^{2}-q+1$. In the first case, $q^{2}+1$ is divisible by 5 , a contradiction. In the second case, resp. the third case, choose a primitive prime divisor $\ell$ of $q^{12}-1$, resp. of $q^{6}-1$; cf. [Zs. Observe that $13 \mid 2^{12}-1$, $41 \mid 2^{20}-1$ and $6 f \geq 18$. Hence the primitivity of $\ell$ implies that $\ell \neq 2,3,13,41$. But clearly $\ell$ divides $\chi(1)$. Thus $\chi(1)$ cannot be written in the form $2^{a} 3^{b} 13^{c} 41^{d}$, again a contradiction.

2) Next let $G=F_{4}(q)$. Choose a primitive prime divisor $\ell$ of $q^{8}-1$. If $q>2$, then let $\ell^{\prime}$ be a primitive prime divisor of $q^{12}-1$. If $q>4$, then let $\ell^{\prime \prime}$ be a primitive prime divisor of $q^{6}-1$. By the choice, $\ell, \ell^{\prime}, \ell^{\prime \prime} \neq 2,3,13,41$.

Assume that $\chi$ is a unipotent character. Then $\chi(1)$ is listed in [C2, p. 479]. Since $\ell$ divides $q^{4}+1, \chi(1)$ is not divisible by $q^{4}+1$. Since $\ell^{\prime}$ divides $q^{4}-q^{2}+1$ if $q>2$ and $q^{2}+q+1=7$ if $q=2, \chi(1)$ cannot be divisible by $\left(q^{4}-q^{2}+1\right)\left(q^{2}+q+1\right)$. These two conditions exclude all but three unipotent characters, $F_{4}[ \pm i]$ and $\phi_{16,5}$ in the notation of [C2]. The first two characters have degree $d:=\frac{1}{4} q^{4}\left(q^{2}-1\right)^{4}\left(q^{6}-1\right)^{2}$. It is easy to see that $d$ is divisible by 7 if $q=2$, by 5 if $q=4$, and by $\ell^{\prime \prime}$ if $q>4$. Similarly, the degree of the character $\phi_{16,5}$ is divisible by 5 if $q=2$ and by $\ell^{\prime}$ if $q>2$. In any of these cases we arrive at a contradiction.

Finally, we assume that $\chi$ is not a unipotent character. Then, according to Luzstig's classification of irreducible characters of $G, \chi(1)$ is divisible by $e:=$ $\left(G: C_{G}(s)\right)_{2^{\prime}}$ for some nontrivial semisimple element $s \in G$, since the group $G$ is self-dual. The order of $C_{G}(s)$ is given in [Sh1, Table III]. In particular, in all but one case $e$ is divisible by $q^{4}+1$ and so by $\ell$, a contradiction. In the only exception, $e$ is divisible by $q^{12}-1$ and so by 7 , again a contradiction.

Remark 3.4. Let $G={ }^{2} F_{4}(2)^{\prime}$ be the Tits simple group. Then $\chi \in \operatorname{Irr}(G)$ is irreducible modulo 2 precisely when $\chi$ is one of the following characters: the trivial character, one of the two characters of degree 26 (these two characters are globally irreducible), and one of the two characters of degree 2048. This can be seen by inspecting [Atlas] and [JLPW].

Remark 3.5. Let $q=2^{f}>2$ and let $G={ }^{2} B_{2}(q)$. Suppose $\chi \in \operatorname{Irr}(G)$ is irreducible modulo 2. Then the conclusion of Proposition [3.3] also holds for $\chi$, since the degrees of irreducible 2-modular characters of $G$ are 2-powers, meanwhile the only nontrivial irreducible complex character of 2-power degree of $G$ is the Steinberg character; cf. $\underline{\mathrm{Su}}$. If $q=8$, then the Schur multiplier of $G$ is $\mathbb{Z}_{2}^{2}$, and there are 3 characters of degree 64 , one for each central extension $2 G$, that are irreducible modulo 2 .

Proposition 3.6. Let $G={ }^{3} D_{4}(q)$ with $q=2^{f}$. Suppose that a complex irreducible character $\chi$ of $G$ is irreducible modulo 2. Then either $\chi$ is the trivial character, or $\chi$ is the Steinberg character, or $q=2$ and $\chi$ is the unique irreducible character of $G$ of degree 26.

Proof. Assume the contrary: $\chi(\bmod 2)$ is irreducible, but $\chi$ is neither the trivial character nor the Steinberg character. The case $q=2$ can easily be checked using [Atlas] and [JLPW], therefore we may assume that $q>2$. According to [JLPW], the degree of any irreducible 2-modular character of $D_{4}(2)$ is either $1,8,26,160$, 246,784 , and 2048 , and so it can be divisible only by the primes $2,3,5,7,13$, 
and 41. By our assumption and by Steinberg's Tensor Product Theorem, the same thing is true for $\chi(1)$.

Choose a primitive prime divisor $\ell$ of $q^{12}-1$. Then clearly $\ell \neq 2,3,5,7,13,41$. Since $\ell \mid\left(q^{4}-q^{2}+1\right), \chi(1)$ cannot be divisible by $q^{4}-q^{2}+1$. Inspecting character degrees of $G$ as listed in $\overline{\mathrm{DM}}$, we see that $\chi$ can be only one of three characters, $\rho_{1}$ of degree $\frac{1}{2} q^{3}\left(q^{3}+1\right)^{2}, \chi_{14}$ of degree $\left(q^{6}-1\right)^{2}$, or ${ }^{3} D_{4}[-1]$ of degree $\frac{1}{2} q^{3}\left(q^{3}-1\right)^{2}$ (in the notation of [DM]). If $f \geq 3$, then we can exclude $\rho_{1}$ and $\chi_{14}$ by using a primitive prime divisor of $q^{6}-1$. If $f \geq 5$ or $f=3$, then we can exclude ${ }^{3} D_{4}[-1]$ by using a primitive prime divisor of $q^{3}-1$. If $f=2$, then one can check directly that the degree of $\rho_{1}, \chi_{14}$, and ${ }^{3} D_{4}[-1]$ cannot be written as a product of the degrees of two irreducible 2-modular characters of $D_{4}(2)$. Finally, if $f=4$, then one directly checks that the degree of ${ }^{3} D_{4}[-1]$ cannot be written as a product of the degrees of four irreducible 2-modular characters of $D_{4}(2)$.

Proof of Theorem 1.2. It follows directly from Propositions 3.1, 3.3, and 3.6 and Remark 3.5 The case of $G_{2}(q)$, where $q=p^{f}$ and $p>3$, has already been handled in [TZ1] and [TZ2].

Some of the exceptions mentioned in Remarks 3.2 and 3.5 are explained by the nontrivial $p$-part of the Schur multiplier as follows. Let $G$ be a group with a nontrivial center $Z$. Let $p$ be a prime dividing $|Z|$. Denote by $P$ the Sylow $p$ subgroup of $Z$ and set $H=G / P$. Let $\Phi_{H}$ be a complex irreducible representation of $H$ of degree $d$ and of $p$-defect 0 . Let $\Phi$ denote the inflation of $\Phi_{H}$ to $G$, and let $B$ be the block of $G$ containing $\Phi$.

Proposition 3.7. B contains exactly $|Z|_{p}$ complex irreducible representations of $G$. The degree of each of them is equal to $d$. Moreover, they have the same reduction modulo $p$, which is an irreducible representation of $G$ with kernel $P$.

Proof. Let $D$ be the defect group of $B$. Then $D=P$ by [Do, Lem. 64.3]. Now the proposition is exactly [F Thm. V.4.7], except for the last claim which follows from [F, Cor. V.4.6].

Corollary 3.8. Let $H$ be a simple group of Lie type in characteristic $p$ and $\Phi$ be its Steinberg representation. Suppose that the order of the Schur multiplier of $H$, $|\operatorname{Mult}(H)|$, is divisible by $p$, and let $p^{a}=|\operatorname{Mult}(H)|_{p}$. Let $\hat{H}$ be the covering group of $H$ with center of order $p^{a}$. Then $\hat{H}$ has exactly $p^{a}$ nonequivalent complex irreducible representations of degree $|H|_{p}$, and the reduction modulo $p$ of all of them is the same irreducible representation of $H$.

\section{REFERENCES}

[Atlas] J. H. Conway, R. T. Curtis, S. P. Norton, R. A. Parker and R. A. Wilson, 'An ATLAS of Finite Groups', Clarendon Press, Oxford, 1985. MR 88g:20025

[B] A. Borel, R. Carter, C. W. Curtis, N. Iwahori, T. A. Springer, R. Steinberg, 'Seminar on Algebraic Groups and Related Finite Groups', Lect. Notes in Math., vol. 131, SpringerVerlag, Berlin, 1970. MR 41:3486

[C1] R. W. Carter, Centralizers of semisimple elements in the finite classical groups, Proc. London Math. Soc. 42 (1981), 1 - 41. MR 82c:20040

[C2] R. Carter, 'Finite Groups of Lie type: Conjugacy Classes and Complex Characters', Wiley, Chichester, 1985. MR 87d:20060 reprint MR 90g:20001

[CR] B. Chang and R. Ree, The characters of $G_{2}(q)$, Symp. Math. 13 (1974), 395 - 413. MR 51:673 
[D] D. I. Deriziotis, The centralizers of semisimple elements of the Chevalley groups $E_{7}$ and $E_{8}$, Tokyo J. Math. 6 (1983), 191 - 216. MR 85g:20060

[DL] D. I. Deriziotis and M. W. Liebeck, Centralizers of semisimple elements in finite twisted groups of Lie type, J. London Math. Soc. 31 (1985), 48 - 54. MR 87e:20087

$[\mathrm{DM}] \quad$ D. I. Deriziotis and G. O. Michler, Character table and blocks of finite simple triality groups ${ }^{3} D_{4}(q)$, Trans. Amer. Math. Soc. 303 (1987), $39-70$. MR 88j:20011

[DiM] F. Digne and J. Michel, 'Representations of Finite Groups of Lie Type', London Mathematical Society Student Texts 21, Cambridge University Press, 1991. MR 92g:20063

[Do] L. Dornhoff, 'Group Representation Theory', Marcel Dekker, New York, 1972. MR 50:458b

[E] H. Enomoto, The characters of $G_{2}(q), q=3^{f}$, Japan. J. Math. 2 (1976), $191-248$. MR 55:10552

[EY] H. Enomoto and H. Yamada, The characters of $G_{2}\left(2^{n}\right)$, Japan. J. Math. 12 (1986), 325 - 377. MR 89d:20006

[F] W. Feit, 'The Representation Theory of Finite Groups', North-Holland, Amsterdam, 1982. MR 83g:20001

[GLS] D. Gorenstein, R. Lyons, and R. Solomon, 'The classification of the finite simple groups. Number 3. Part I. Chapter A. Almost simple K-groups', Mathematical Surveys and Monographs, 40.3. American Mathematical Society, Providence, RI, 1998. MR 98j:20011

[Gr] B. H. Gross, Group representations and lattices, J. Amer. Math. Soc. 3 (1990), 929-960. MR 92a:11077

$[J] \quad$ J. C. Jantzen, Representations of Chevalley groups in their own characteristic, Proc. Symp. Pure Math. vol. 47 (1987), pt. 1, 127 - 146. MR 89g:20076

[JLPW] C. Jansen, K. Lux, R. A. Parker and R. A. Wilson, 'An Atlas of Brauer Characters', Oxford University Press, Oxford, 1995. MR 96k:20016

[L] G. Lusztig, 'Characters of reductive groups over a finite field', Annals of Math. Studies 107, Princeton Univ. Press, Princeton, 1984. MR 86j:20038

[Sh1] K. Shinoda, The conjugacy classes of Chevalley groups of type $\left(F_{4}\right)$ over finite fields of characteristic 2, J. Fac. Sci. Univ. Tokyo Sect. IA Math. 21 (1974), 133 - 159. MR 50:2356

[Sh2] K. Shinoda, The conjugacy classes of the finite Ree groups of type $F_{4}$, J. Fac. Sci. Univ. Tokyo Sect. IA Math. 22 (1975), 1-15. MR 51:8281

[Sho] T. Shoji, The conjugacy classes of Chevalley groups of type $\left(F_{4}\right)$ over finite fields of characteristic $p \neq 2$, J. Fac. Sci. Univ. Tokyo Sect. IA Math. 21 (1974), $1-17$. MR 50:10109

[St] R. Steinberg, 'Lectures on Chevalley Groups', Yale Univ., 1967. MR 57:6215

[Su] M. Suzuki, On a class of doubly transitive groups, Ann. of Math. 75 (1962), $105-145$. MR 25:112

[T] J. G. Thompson, Finite groups and even lattices, J. Algebra 38 (1976), 523 - 524. MR 53:3108

[TZ1] Pham Huu Tiep and A. E. Zalesskii, Mod $p$ reducibility of unramified representations of finite groups of Lie type, Proc. London Math. Soc. 84 (2002), 343-374.

[TZ2] Pham Huu Tiep and A. E. Zalesskii, Strong rationality of unipotent elements and realization fields of complex representations of finite groups of Lie type (submitted).

[V] F. D. Veldkamp, Representations of algebraic groups of type $F_{4}$ in characteristic $2, J$. Algebra 16 (1970), 326 - 339. MR 42:4651

[Zs] K. Zsigmondy, Zur Theorie der Potenzreste, Monath. Math. Phys. 3 (1892), 265 - 284.

Department of Mathematics, University of Florida, Gainesville, Florida 32611-8105

E-mail address: tiep@math.ufl.edu

School of Mathematics, University of East Anglia, Norwich NR4 7TJ, United KingDOM

E-mail address: a.zalesskii@uea.ac.uk 